\title{
Muon reconstruction and performances with the ATLAS detector
}

\author{
Federico Sforza*, on behalf of the ATLAS Collaboration. \\ Max-Planck-Institut für Physik \\ E-mail: federico.sforza@cern.ch
}

\begin{abstract}
The ATLAS detector is designed for efficient muon identification and high precision momentum measurement across three orders of magnitude of muon energy (from a few $\mathrm{GeV}$ up to a few $\mathrm{TeV}$ ). This is possible thanks to two high precision tracking systems, the Inner Detector and the Muon Spectrometer, which provide independent momentum measurements that can be successively combined.

Reconstruction performances are measured with data driven techniques using the $J / \psi \rightarrow \mu \mu$ and $Z \rightarrow \mu \mu$ decays produced in more than $20 \mathrm{fb}^{-1}$ of $p-p$ collisions. The muon simulation is constantly compared to the measured performances and specific corrections on efficiency, momentum resolution and scale are applied when needed.
\end{abstract}

14th International Conference on B-Physics at Hadron Machines, April 8-12, 2013

Bologna, Italy

${ }^{*}$ Speaker. 


\section{Introduction}

The ATLAS experiment [1] is a multipurpose detector designed for the investigation of the physics processes originating from the $p-p$ collisions produced at the Large Hadron Collider. During the 2011 and the 2012 data taking periods (with center of mass energies of 7 and $8 \mathrm{TeV}$ respectively), the muon identification and reconstruction systems played a relevant role both in searches for new physics, Higgs physics studies, and in precision measurements of known Standard Model processes.

Two main sub-detector systems are involved in muon identification and reconstruction: the Muon Spectrometer [2] (MS) and the Inner Detector [3] (ID). They provide independent momentum measurements that can be successively combined with the use of specific algorithms to increase the purity and performances of the reconstructed muon. This allows optimal performance over three orders of magnitude of muon energy: from a few $\mathrm{GeV}$ (needed for $B$-physics studies) up to a few $\mathrm{TeV}$ (where new physics may appear).

This paper briefly describes the algorithms used for the muon identification and reconstruction in the ATLAS experiment. Their performance, namely the efficiency and the momentum resolution, are measured using data-driven techniques that, in general, involve the reconstruction of $Z \rightarrow \mu \mu$ or $J / \psi \rightarrow \mu \mu$ decays. The muon Monte Carlo simulation is also compared to data so that specific corrections may be derived.

\section{Muon Detection and Reconstruction}

The MS is the largest of all ATLAS sub-detectors and it is designed to detect tracks ${ }^{1}$ in the region $0<|\eta|<2.7$ and to provide momentum measurement with a relative resolution from $3 \%$ to $10 \%$ (at $p_{T}=1 \mathrm{TeV}$ ). The MS is built by one barrel $(|\eta|<1.05$ ) and two endcaps sections within a toroidal field of about $0.5 \mathrm{~T}$. Triggering and $\eta, \phi$ position measurements, with approximately $1 \mathrm{~cm}$ resolution, are provided by the Resistive Plate Chambers (RPC, three layers for $|\eta|<1.05$ ) and by the Thin Gap Chambers (TGC, three layers for $2.0<|\eta|<2.5$ ). While precision momentum measurement in the transverse plane is provided by the Monitored Drift Tube Chambers (MDT, three layers for $|\eta|<2.0$, two layers for $2.0<|\eta|<2.5$ ) and the Cathode Strip Chambers (CSC, $2.0<|\eta|<2.7$ ) thanks to their hit resolution of, respectively, $\approx 80 \mu \mathrm{m}$ and $\approx 60 \mu \mathrm{m}$ in the bending $(\eta)$ plane.

An additional determination of the muon momentum is provided by the ID. This consists of high-resolution sub-detectors at the inner radii and continuous tracking elements at the outer radii, all contained in a solenoidal magnetic field of 2 T. In particular there is an inner Silicon Pixels and Semi-Conductor Tracker (SCT) detector for $|\eta|<2.5$ and an outer Transition Radiation Tracker (TRT) covering $|\eta|<2.0$.

Muon identification is performed according to several reconstruction criteria (also named "types"), according to the available information from the ID and the MS sub-detector systems. The different types are:

\footnotetext{
${ }^{1}$ A system of cylindrical coordinates is used, with the $z$ axis along beam-pipe, azimuthal angle $\phi$ and polar angle $\theta$. The transverse momentum $p_{T}=p \sin \theta$ and the pseudo-rapidity $\eta=-\ln [\tan (\theta / 2)]$ are also defined.
} 
- Combined (CB): in this case the ID and MS track measurements are combined (for $|\eta| \lesssim 2.65$ ). The combination improves the resolution with respect to the single ID and MS measurements (in a range $6<p_{T}<100 \mathrm{GeV} / c$ ) and it allows rejection of muons from secondary interactions or from $\pi / K$ decays in flight. The CB reconstruction is performed using several complementary strategies (named "chains"): in particular it is possible to combine statistically the track parameters and covariance matrices measured in the ID and in the MS or it is possible to perform a global re-fitting of the muon track using the hits from both the ID and MS sub-detectors. The CB type is considered the main muon reconstruction algorithm because of the its good efficiency and momentum resolution.

- Segment-tagged: the muon is identified only by segments in the MS, so the momentum can be reconstructed only using the ID information. This provides uniform coverage (for $|\eta|<2.5$ ) also for low momentum tracks. Momentum resolution is good in the $3<p_{T}<50$ $\mathrm{GeV} / c$ range.

- Stand-alone: the muon is reconstructed using only the MS information. This extends the coverage up to $|\eta|=2.7$. Resolution is good for high $p_{T}$ tracks because of the large lever arm.

- Calorimeter-tagged: the muon is identified only by a track in the ID and by calorimetric information. This type has the lower purity but it recovers acceptance in the un-instrumented region of the MS for $\eta \approx 0$.

\section{Performances: Reconstruction Efficiency}

The performance of the muon system is constantly monitored comparing the ATLAS simulation to known physics processes selected in data. The first important parameter, described in this section, is the muon reconstruction efficiency.

The "tag-and-probe" method [4] uses the complementarity of the MS and ID track measurements to evaluate the total and partial reconstruction efficiencies for the different muon types. The method requires the selection of a di-muon decay process, for example $Z \rightarrow \mu \mu$ decays, in which one of the muons, named "tag", is reconstructed in both systems and the other, named "probe", is identified by just one of the subsystems in order to probe the reconstruction efficiency of the other. If a CB muons is used as a tag, the ID reconstruction efficiency can be measured with a stand-alone muon probe. Vice-versa, in the case of MS reconstruction efficiency or matching efficiency measurements, the probe is a calorimeter-tagged muon. The total $\mathrm{CB}$ reconstruction efficiency is then derived from product of the ID, the MS and the matching efficiencies.

The measured efficiency is compared to the one predicted from simulation and, if needed, a data-MC Scale Factor (SF) correction is derived to be used in other ATLAS analyses. The SF correction itself (for the high statistics 2012 dataset) ranges from $0.2 \%$ to $0.5 \%$ with systematic uncertainties of the order of 0.1 to $1 \%$ in a wide range of $p_{T}$. The uncertianty for $p_{T}<7 \mathrm{GeV} / c$ rises to $2 \%$ according to $J / \psi \rightarrow \mu \mu$ tag-and-probe measurements [5].

The very large statistics of $Z \rightarrow \mu \mu$ decays recorded in the 2011 and 2012 data taking allows the detailed study of the reconstruction efficiency against several variables of interest. For example 
Figure 1 shows the reconstruction efficiency for CB plus Segment-tagged muons as a function of the muon $\eta$ and of the average number of inelastic $p p$ collisions per crossing (the $\langle\mu\rangle$ parameter), displaying high value (in average above 97\%) and remarkable stability.
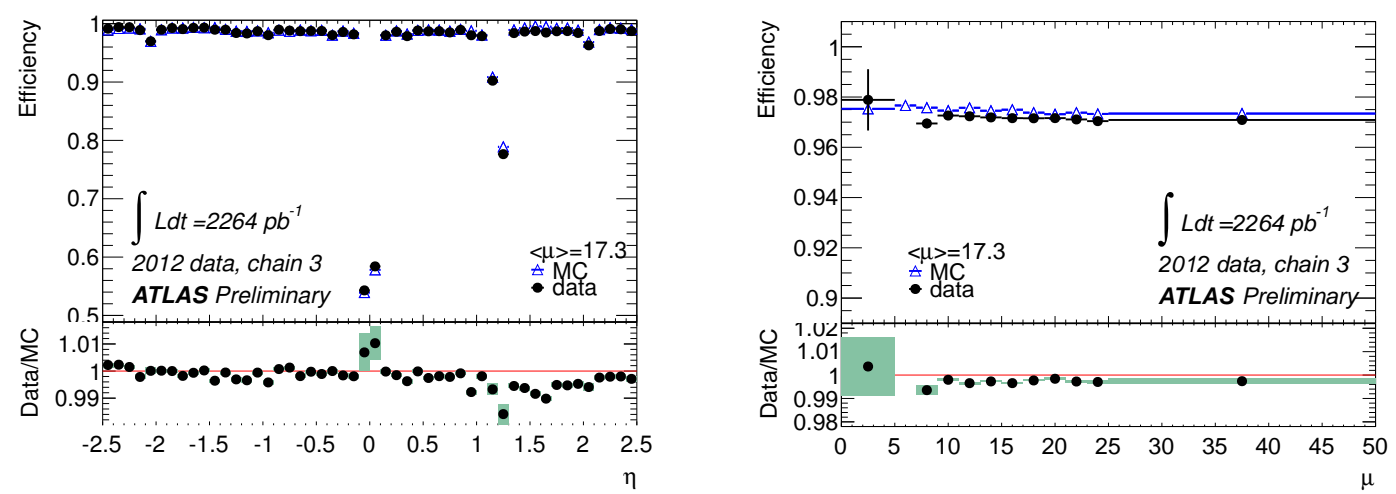

Figure 1: Reconstruction efficiency [6] for CB muons (left plot) and CB plus Segment-Tagged muons (right plot) versus the muon $\eta$ and $\langle\mu\rangle$ distributions. Un-instrumented regions of the MS produce the efficiency drops at $\eta \approx 0$ and at $\eta \approx 1.2$.

\section{Performances: Momentum Resolution and Scale}

The momentum resolution is another important parameter used in the evaluation of the muon reconstruction system performance. Here the decay in di-muons of the $Z$ and $J / \psi$ resonances in data are used as standard candles for the precise calibration of the muon momentum simulation (both scale and resolution). The corrected MC can be used for the evaluation of the muon resolution itself and for successive and independent mass or momentum measurements.

In the correction procedure [7], the simulated transverse momentum $\left(p_{T}^{M C}\right)$ is multiplied by scale (Scale) and smearing terms $(G(\mu, \sigma)$ is a Gaussian random variable of mean $\mu$ and sigma $\sigma$ ):

$$
p_{T}^{\prime}=p_{T}^{M C} \cdot \operatorname{Scale}\left(1+\Delta p_{1} G(0,1)+\Delta p_{2} G(0,1) p_{T}\right),
$$

where the $\Delta p_{1}$ and $\Delta p_{2}$ parameters are interpreted, respectively, as a correction to the multiple scattering and to the alignment of the simulation. A template fit of the $Z \rightarrow \mu \mu$ data line-shape is used to derive the Scale correction for both the MS and the ID sub-detectors, the $\Delta p_{1}$ term for the MS, and the $\Delta p_{2}$ term for the ID. The remaining parameters, to which the fit is not sensitive, are covered by systematic variations.

Figure 2 shows the $Z \rightarrow \mu \mu$ line-shape for data corresponding to the complete 2012 dataset of $20.7 \mathrm{fb}-1$ and for the Powheg [8] simulation before and after the scale and resolution corrections derived from the same data set. Although corrections are on the order of the $0.1 \div 0.2 \%$, the effect is noticeable given the very large statistics of $Z \rightarrow \mu \mu$ events, corresponding to more than $4 \cdot 10^{6}$ selected events. The final resolution (defined as the width of a Gaussian convoluted with the di-muon mass distribution at generator level) ranges from 1.5 to $3 \mathrm{GeV} / c^{2}$ at $M_{Z}$ in the different detector regions. 

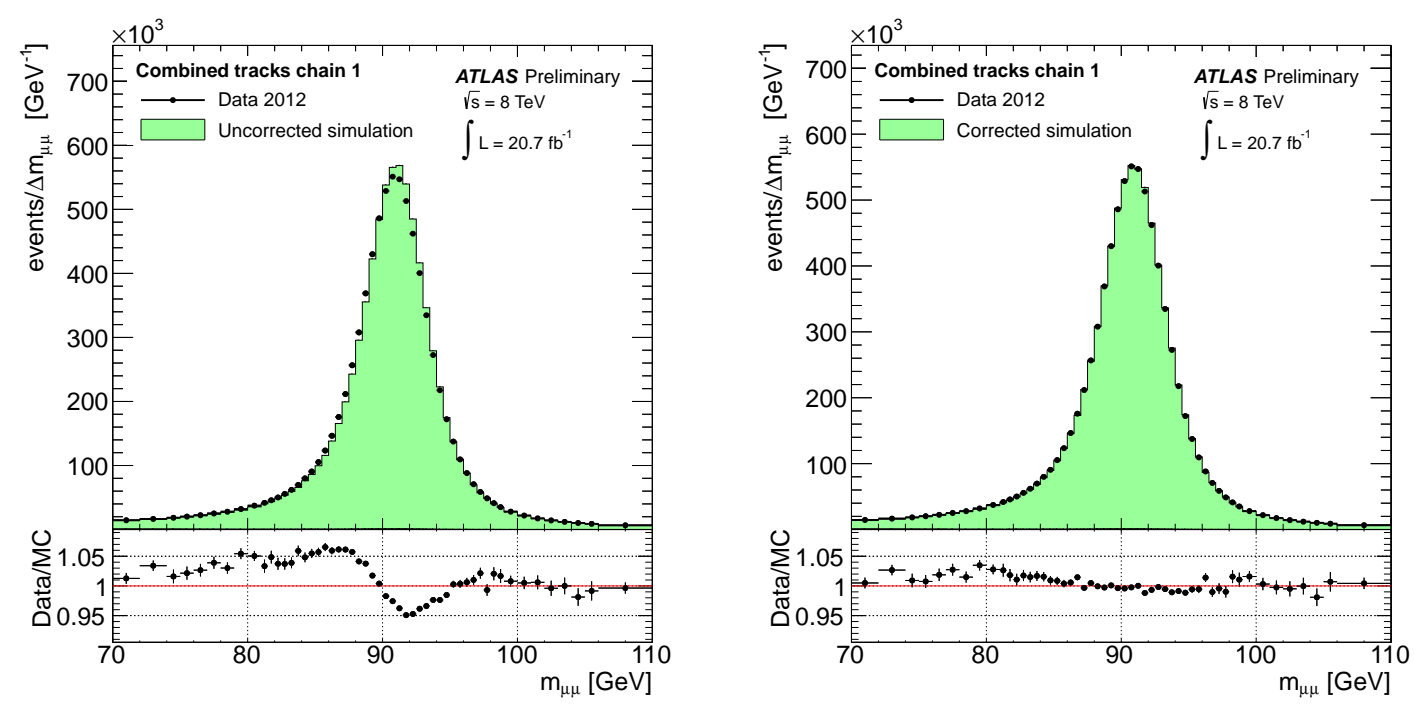

Figure 2: Di-muon invariant mass for chain 1, CB muons, isolated and with pT $>25 \mathrm{GeV}$ [6]. The plot shows the invariant mass for 2012 data and corrected Powheg [8] simulation of $Z \rightarrow \mu \mu+$ backgrounds. No corrections is applied on the left plot while smearing and scale correction are applied to the plot on the right. The corrections have been derived from the full 2012 dataset.

Given the importance of the scale correction for independent measurements, it has been checked also with other physics process $(J / \psi, \Upsilon)$ resulting in a consistent behaviour across a wide range of $p_{T}$, as shown in Figure 3.

\section{Conclusions}

This paper summarizes the muon reconstruction algorithms used in the ATLAS experiment and their performance as measured in data recoded during the 2011 and $2012 p p$ collisions at 7 and $8 \mathrm{TeV}$ of center of mass energy, respectivelly. The reconstruction types are classified according to the information available in the ID and in the MS independent tracking systems. Muon reconstruction efficiency, measured with the tag-and-probe method, is high (in avarage above 97\%) and well reproduced by the simulation when analyzed against several variable of interest. The momentum resolution, analyzed with a template fit of the $Z \rightarrow \mu \mu$ decay line-shape, ranges from 1.5 to $3 \mathrm{GeV} / c^{2}$ at $M_{Z}$ in the different detector regions. Both momentum resolution and scale appear to be well reproduced by the simulation, with only a small correction derived from the $Z \rightarrow \mu \mu$ fit and from analyses of the $J / \psi \rightarrow \mu \mu$ decay.

\section{References}

[1] ATLAS Collaboration, The ATLAS Experiment at the CERN Large Hadron Collider, JINST 3 (2008) S08003.

[2] ATLAS Collaboration, Commissioning of the ATLAS Muon Spectrometer with Cosmic Rays, Eur.Phys.J. C 70, (2010) 875. 


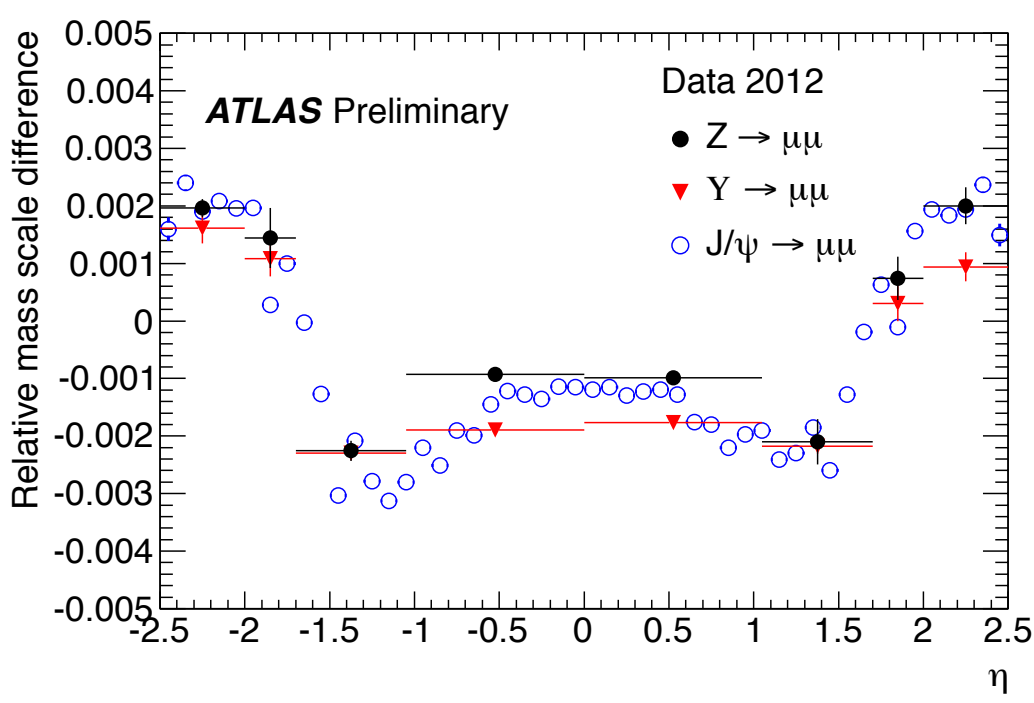

Figure 3: Relative mass scale $\left(m_{\mu \mu}-m_{P D G}\right) / m_{P D G}$ for $Z, \Upsilon$ and $J / \psi$ in 2012 data collected with the ATLAS detector [6]. Selected $Z$ events require two CB muons with $p_{T}>25 \mathrm{GeV} / c$ and same $\eta$ bin, selected $\Upsilon$ events require two CB muons of $p_{T}>6 \mathrm{GeV} / \mathrm{c}$ and same $\eta$ bin, selected $J / \psi$ events require two $\mathrm{CB}$ muons of $p_{T}>6 \mathrm{GeV} / \mathrm{c}$ and the $\eta$ of the leading muon is shown. The mass scale obtained for the three different mass points is consistent.

[3] ATLAS Collaboration, The ATLAS Inner Detector commissioning and calibration, Eur.Phys.J. C 70, (2010) 787.

[4] ATLAS Collaboration, Muon reconstruction efficiency in reprocessed 2010 LHC proton-proton collision data recorded with the ATLAS detector, ATLAS-CONF-2011-063 (2011).

[5] ATLAS Collaboration, A measurement of the muon reconstruction efficiency in 2010 ATLAS data using $J / \psi$ decays, ATLAS-CONF-2012-125 (2012).

[6] ATLAS Collaboration, Preliminary Figures from 2012 Collision Data at $8 \mathrm{TeV}$ (2012):

https://twiki.cern.ch/twiki/bin/view/AtlasPublic/MuonPerformancePublicPlots

[7] ATLAS Collaboration, Muon Momentum Resolution in First Pass Reconstruction of pp Collision Data Recorded by ATLAS in 2010, ATLAS-CONF-2011-046 (2011).

[8] Alioli S. et al., A general framework for implementing NLO calculations in shower Monte Carlo programs: the POWHEG BOX, JHEP 1006 (2010) 43. 